manner; no peptide, $2468.5 \pm \mu \mathrm{g} / \mathrm{ml}, 5 \mathrm{mg}, 2181.7 \pm 26.2 \mathrm{ng} / \mathrm{ml}$ $(\mathrm{p}=0.006), 10 \mathrm{mg}, 1576 \pm 164.7 \mathrm{ng} / \mathrm{ml}(\mathrm{p}=0.001)$, and 20 and $30 \mathrm{mg}$ completely prevented polymer formation in inclusions $(p \leq 0.001)$. Unrelated peptides had no effect. Elastase activity of AT in the supernatant from Z-AT cells was significantly reduced compared to M-AT cells; $p \leq 0.001$, in keeping with the secretory defect due to retention of Z-AT in inclusion bodies. The elastase activity (and AT concentration) in the supernatant from Z-AT cells was restored by $20 \mathrm{mg} 4 \mathrm{M}$, O.D. $405 \mathrm{~nm}$, Z-AT vs Z-AT + $20 \mu \mathrm{g} 4 \mathrm{M}, 0.129 \pm 0.009$ vs $0.788 \pm 0.054$ respectively, $(p \leq 0.001)$, where a higher O.D. represents higher elastase activity. Functional activity of secreted AT following treatment with $4 \mathrm{M}$ was confirmed by its ability to form an SDS-stable complex with elastase as shown by immunoblot. RTPCR showed that the ER accumulation of Z-AT induced cell stress; NF- $\kappa \mathrm{B}$ activation, expression of protein kinase RNA (PKR)-like ER kinase (PERK), and IL-6 $(100.4 \pm 16 \mathrm{pg} / \mathrm{ml})$ and IL-8 $(2592.5 \pm 575 \mathrm{pg} /$ $\mathrm{ml}$ ), all of which could be abrogated effectively by $20 \mathrm{mg} 4 \mathrm{M}$ (IL-6, $45.8 \pm 28 \mathrm{pg} / \mathrm{ml}, \mathrm{p} \leq 0.001$ and IL-8, $184.3 \pm 29 \mathrm{pg} / \mathrm{ml}, \mathrm{p}=0.014)$. These findings are the first evidence that inhibitors of Z-AT polymerisation targeting s4A can prevent its cellular accumulation and deleterious effects. Importantly, this strategy was also able to improve plasma concentration of Z-AT.

\section{S52 ASSOCIATION OF MICROTUBULE INSTABILITY WITH DEFECTIVE PHAGOCYTOSIS IN COPD}

doi:10.1136/thoraxjnl-2011-201054b.52

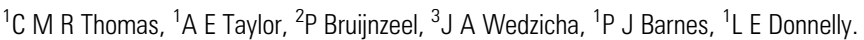
${ }^{1} \mathrm{NHLI}$, Imperial College, London, UK; ${ }^{2}$ Astra Zeneca R\&D, Charnwood, UK; ${ }^{3} \mathrm{UCL}$ Medical school, London, UK

Acute exacerbations of COPD are the commonest cause of acute medical admissions in the UK and $\sim 50 \%$ are associated with bacterial infection. Alveolar macrophages (AM) normally clear inhaled bacteria but defective phagocytosis may lead to chronic colonisation and increased exacerbations. Monocyte-derived macrophages (MDM), used to model AM, were obtained from COPD, smoking and healthy subjects. MDM phagocytosis of fluorescently-labelled polystyrene beads, Haemophilus influenzae (HI) or Streptococcus pneumoniae (SP) was measured by fluorimetry. MDM derived from all subjects showed equivalent ability to phagocytose beads, however, COPD and smoker MDM showed significantly reduced phagocytosis of bacteria. Phagocytosis of $\mathrm{HI}$ was reduced by $28 \%$ and $48 \%$ in COPD and smoker MDM respectively, compared to healthy, while SP phagocytosis was reduced by $32 \%$ and $52 \%$ in COPD and smoker MDM respectively, compared to healthy (Abstract S52 table 1). Having identified defective bacterial phagocytosis in smoker and COPD MDM, the next step was to elucidate the underlying mechanism. Cytoskeletal rearrangement was investigated, with COPD MDM showing significantly reduced phagocytosis of bacteria in comparison to healthy after pre-incubation with nocodazole (microtubule disruptor). Microtubules are involved in membrane trafficking of the phagolysosome and microtubule stability is necessary for effective phagocytosis. Tubulin is acetylated to form stable microtubules and is deacetylated by HDAC6 and Sirt2. COPD MDM showed reduced levels of acetylated tubulin compared to healthy MDM. Pre-incubation with epothilone B $(10 \mathrm{~nm})$ a microtubule stabiliser, improved $\mathrm{HI}$ phagocytosis in

Abstract S52 Table 1 Relative fluorescence values $\left(\mathrm{RFU} \times 10^{3}\right)$ for MDM phagocytosis assays at $4 \mathrm{~h}$

\begin{tabular}{lll}
\hline & HI & SP \\
\hline Healthy $(n=21)$ & $11.5 \pm 0.9 \mathrm{RFU} \times 10^{3}$ & $8.2 \pm 1.4 \mathrm{RFU} \times 10^{3}$ \\
Smoker $(\mathrm{n}=20)$ & $4.6 \pm 0.6(\mathrm{p}<0.001)$ & $3.7 \pm 0.6 \quad(\mathrm{p}<0.001)$ \\
COPD $(n=23)$ & $8.4 \pm 1(p<0.01)$ & $5.7 \pm 0.7(p<0.05)$ \\
\hline
\end{tabular}

COPD MDM by $20 \%(\mathrm{p}<0.05)$ and SP phagocytosis in smoker MDM by $40 \%$. Levels of acetylated tubulin increased on exposure to bacteria alone in healthy and smoker MDM but not in COPD MDM. Pre-incubation with epothilone $B$ was associated with significantly increased levels of acetylated tubulin in COPD cells. No significant differences were seen in the expression of HDAC6 or Sirt2 in COPD compared to healthy cells. MDM from smoking and COPD subjects show reduced phagocytosis of common respiratory bacterial pathogens. Acetylation of microtubules appears to be reduced in COPD, whereas, increasing tubulin acetylation is associated with improvements in phagocytosis, which may allow for targeted development of future therapies to treat colonisation and prevent exacerbations of COPD.

\section{S53 ALARMINS IN BRONCHIOLITIS OBLITERANS SYNDROME AFTER LUNG TRANSPLANTATION}

doi:10.1136/thoraxjnl-2011-201054b.53

${ }^{1} \mathrm{R}$ Y Mahida, ${ }^{1} \mathrm{M}$ Suwara, ${ }^{2} \mathrm{G}$ Johnson, ${ }^{1} \mathrm{D}$ Mann, ${ }^{2} \mathrm{P}$ A Corris, ${ }^{1} \mathrm{~L}$ Borthwick, ${ }^{2} \mathrm{~A} J$ Fisher. ${ }^{1}$ Tissue Fibrosis and Repair Group, Institute of Cellular Medicine, Newcastle University, Newcastle-Upon-Tyne, UK; ${ }^{2}$ Institute of Transplantation, Freeman Hospital, Newcastle-Upon-Tyne, UK

Introduction Survival after lung transplantation is limited to a median of 5-year due to development of bronchiolitis obliterans syndrome (BOS). BOS is the clinical manifestation of chronic allograft dysfunction, characterised by inflammation and fibrosis of small/medium-sized airways leading to airflow obstruction. Numerous insults to the transplanted lungs have been associated with BOS development. Alarmins are cell derived danger signals released from damaged tissue which activate innate and adaptive immune responses. We hypothesised that the release of alarmins into the airway compartment after lung transplantation may contribute to BOS pathogenesis.

Methods A retrospective longitudinal study of 52 lung transplant recipients from 2005 to present was performed $(26$ recipients developed BOS; 26 remained free from BOS). All recipients had lung function and bronchoalveolar lavage (BAL) performed at 1, 3, 6 and 12 months post transplant. Further samples were taken if the diagnostic criteria for BOS were fulfilled. A total of 214 BAL samples were analysed by ELISA for the alarmins Interleukin-1a (IL-1a) and High Motility Group-Box1 (HMG-B1). Data were analysed using Mann-Whitney tests.

Results Both BOS and non-BOS recipients with culture positive BAL samples had significantly higher concentrations of IL-1a and

\section{IL-1a Assay on Culture Negative BAL Samples}

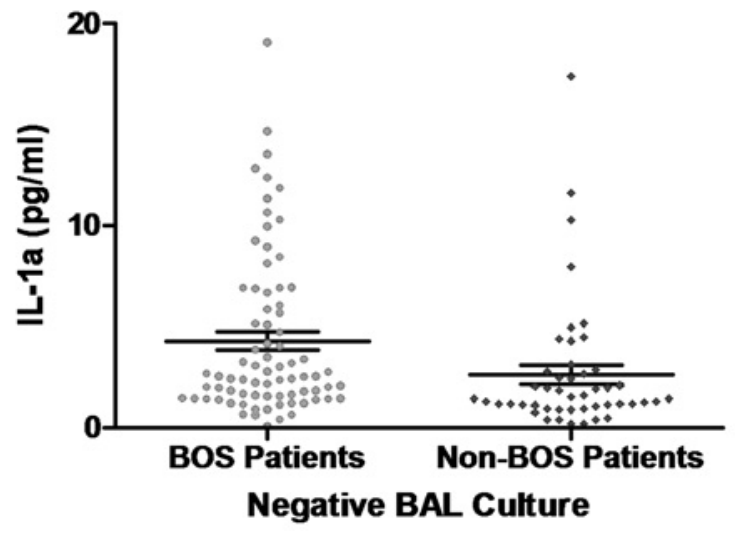

Mann-Whitney $\cup$ Test: $p=0.001$

Abstract S53 Figure 1 IL-1a assay on culture negative BAL samples. 